\title{
AlN-based BAW resonators with CNT electrodes for gravimetric biosensing
}

\author{
L. García-Gancedo ${ }^{a, *}$, Z. Zhu ${ }^{a}$, E. Iborra ${ }^{b}$, M. Clement ${ }^{b}$, J. Olivares ${ }^{b}$, A.J. Flewitt ${ }^{a}$, W.I. Milne ${ }^{a}$, \\ G.M. Ashley ${ }^{\mathrm{c}}$, J.K. Luo ${ }^{\mathrm{c}}$, X.B. Zhao ${ }^{\mathrm{d}}$, J.R. Lu ${ }^{\mathrm{d}}$ \\ a Electrical Engineering Division, University of Cambridge, Cambridge, United Kingdom \\ ${ }^{\mathrm{b}}$ Grupo de Microsistemas y Materiales Electrónicos, Universidad Politécnica de Madrid, Madrid, Spain \\ ${ }^{\complement}$ IMRI, University of Bolton, Bolton, United Kingdom \\ ${ }^{d}$ School of Physics and Astronomy, University of Manchester, Manchester, United Kingdom
}

\section{A R T I C L E I N F O}

Keywords:

Biosensor

Carbon nanotube (CNT)

Aluminum nit ride (AIN)

Bulk acoustic wave resonator (BAW)

Gravimetric biosensor

\begin{abstract}
A B S T R A C T
Solidly mounted resonators (SMRs) with a top carbon nanotubes (CNTs) surface coating that doubles as an electrode and as a sensing layer have been fabricated. The influence of the CNTs on the frequency response of the resonators was studied by direct comparison to identical devices with a top metallic electrode. It was found that the CNTs introduced significantly less mass load on the resonators and these devices exhibited a greater quality factor, $Q(>2000$, compared to $\sim 1000$ for devices with metal electrodes), which increases the gravimetric sensitivity of the devices by allowing the tracking of smaller frequency shifts. Protein solutions with different concentrations were loaded on the top of the resonators and their responses to mass-load from physically adsorbed coatings were investigated. Results show that resonators using CNTs as the top electrode exhibited a higher frequency change for a given load $\left(\sim 0.25 \mathrm{MHz} \mathrm{cm}^{2} \mathrm{ng}^{-1}\right)$ compared to that of a metal thin film electrode $\left(\sim 0.14 \mathrm{MHz} \mathrm{cm}^{2} \mathrm{ng}^{-1}\right)$, due to the lower mass of the CNT electrodes and their higher active surface area compared to that of a thin film metal electrode. It is therefore concluded that the use of CNT electrodes on resonators for their use as gravimetric biosensors is a significant improvement over metallic electrodes that are normally employed.
\end{abstract}

\section{Introduction}

In the last few years there has been an increased need to develop very sensitive biosensors suitable for real time detection. Although there exist numerous types of biosensors such as optically based sensors [1,2], electrochemical sensors [3,4], cantilever-based detectors [5,6] or acoustic wave sensors [7,8], the acoustic wave technologies have the combined merit of all other biosensors: labelfree, ultra-high sensitivity, small size, electronic signal, easy to use and low-cost.

Bulk acoustic wave (BAW) resonators are the most common type of acoustic wave sensors. These devices consist of a piezoelectric layer sandwiched between two metal electrodes to which a microwave (RF) signal is applied [9]. Standing acoustic waves with a well-defined resonant frequency, $f_{r}$, are generated by the microwave signal due to the inverse piezoelectric effect. The application of an external force on the resonator's surface induces additional stresses on the piezoelectric layer which in turn

* Corresponding author at: Electrical Engineering Division, University of Cambridge, 9 IJ Thomson Avenue, Cambridge CB3 OFA, United Kingdom.

Tel.: +4401223748304

E-mail address: luis.garcia-gancedo@eng.cam.ac.uk (L. García-Gancedo) lowers $f_{r}$, with the magnitude of the frequency shift being a function of the external force applied. By tracking changes in $f_{r}$, mass changes on the resonators (for example due the adsorption of biological samples) can be detected. The total mass bound on the resonator's surface is proportional to the change in $f_{r}$. The magnitude of the frequency shift, $\Delta f_{r}$, for a given mass load is proportional to the square of $f_{r}[10]$, hence resonators working at higher frequencies in theory possess a better sensitivity, as a greater $\Delta f_{r}$ is induced for the same change in mass compared to those with lower $f_{r}$.

The quartz crystal microbalance (QCM) is one of the earliest types of acoustic wave device ever used as biosensor. In 1972, it was utilised for the first time to detect antibodies [11]. QCMs have a mass detection limit of a few nanograms, which is limited by low operation frequency $(5-20 \mathrm{MHz})$ due to the quartz substrate thickness. In this respect, the utilisation of thin film technology to develop film bulk acoustic resonators (FBARs) and solidly mounted resonators (SMRs), extremely high frequency versions QCM with an operating frequency in the range of a few $\mathrm{GHz}$, offers great potential to improve the sensitivity and detection limit of gravimetric sensors. Thin film-based resonators offer the additional advantage of lower fabrication cost due to the employment of microfabrication technologies; small dimensions, which makes them suitable for the fabrication of array of sensors for parallel or multi detection; and are compatible with CMOS device fabrication [12-17]. 


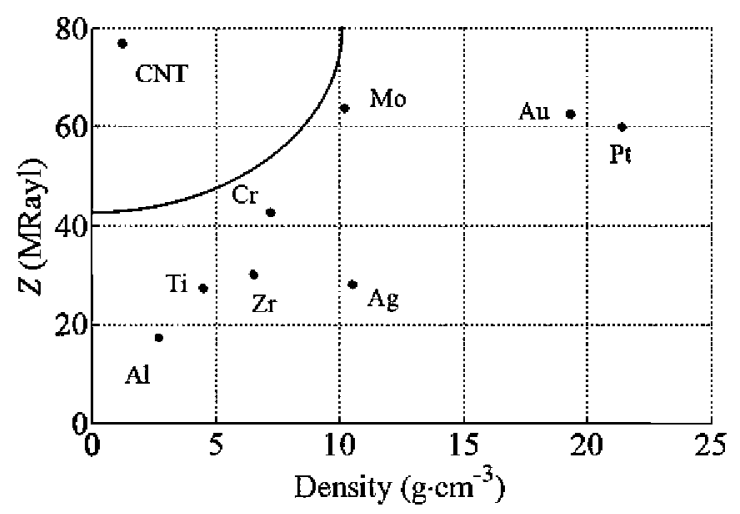

Fig. 1. Density and acoustic impedance of commonly used electrode metals. The best material would be located at the top-left corner, with low mass and high acoustic impedance.

However, higher frequency and smaller dimensions cause all of the environmental and boundary conditions to affect the operation of the resonators more strongly than the QCMs, resulting in a higher noise level and lower quality factors $(Q)$. It is for this reason that, to date, the reported empirical experimentally determined mass sensitivity of thin film-based resonators is not significantly greater than that of QCMs [18-20].

Zinc oxide ( $\mathrm{ZnO}$ ) and aluminum nitride (AIN) thin films are commonly used for the fabrication of BAW resonators due to their good piezoelectric properties which translates into higher $Q$ factors. In the last few years there has been significant work carried out towards the improvement of the structural and electrical properties of $\mathrm{ZnO}$ and AlN thin films for their use as the active material in these devices [18-24] however the $Q$ factors of these resonators are still lower than those of QCMs $[25,26]$, limiting their sensitivity.

Recently, it has also been understood that the material utilised for the electrodes in the resonators plays an important role in their electrical response [24,27-29]. Piezoelectric films are grown on the bottom electrode, which therefore has an influence on the piezoelectric's crystallographic orientation. The electrodes also provide the shape of the resonant area and most importantly, can confine the acoustic energy within the piezoelectric layer. In this scenario, losses are minimised and hence $Q$ is maximised. For gravimetric sensing, the electrode material should have low mass in order to minimise pre-mass damping, high acoustic impedance in order to confine the acoustic waves within the piezoelectric layer and high conductivity in order to minimise the series resistance in the transmission of the excitation signal. The metals most commonly utilised as the electrode materials are aluminum ( $\mathrm{Al}$ ), molybdenum (Mo), tungsten $(\mathrm{W})$, gold $(\mathrm{Au})$ and platinum $(\mathrm{Pt})$, but the low acoustic impedance of $\mathrm{Al}$ and high weight of Mo, W, Au and Pt make all of them less than ideal for their use as the electrode materials.

Carbon nanotubes (CNTs) possess a very high acoustic impedance of $\sim 78 \mathrm{MRayl}$, low densities $\left(\sim 1.5 \mathrm{~g} \mathrm{~cm}^{-3}\right)$, and high electrical conductivities of up to $10^{6} \mathrm{~S} \mathrm{~m}^{-1}$ [30]. Fig. 1 shows a comparison of densities and acoustic impedances of some of the commonly used metals for electrodes, and highlights that CNTs posses the highest acoustic impedance and lowest density, which allows simultaneously low mass electrodes with high acoustic impedance and low series resistance. CNTs have been shown to be an excellent material choice for BAW resonators [29,31-33]. ZnObased FBARs with CNT electrodes directly grown on the substrate were previously fabricated and it was found that the decreased mass associated with the CNTs electrodes increased the base frequency compared to devices with metal electrodes [33]. However the bottom CNTs electrodes cause a significant degradation of the piezoelectric film quality if the film is grown directly on the CNTs

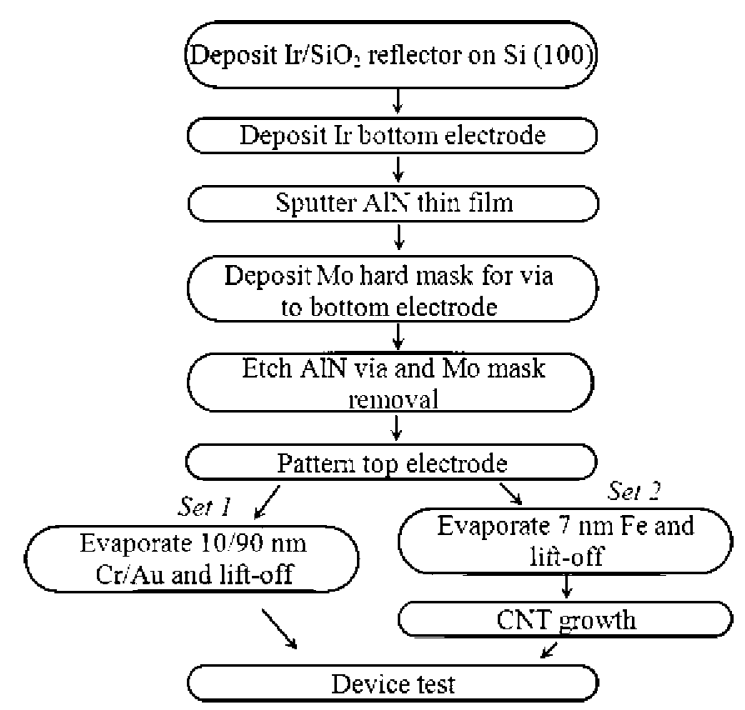

Fig. 2. Fabrication process: main steps.

layer due to the difficulty in growing a piezoelectric layer with good crystallographic orientation on such a rough layer. This may hinder the advantages of a top-CNT electrode alone particularly for biosensing applications [33]. Hence this work investigates the development of SMR devices with a metal bottom electrode that allows excellent piezoelectric film properties, and a top CNTs layer that has a double function as electrode and as a sensing layer.

Biosensors with CNTs sensing electrodes offer an additional benefit compared to those with metal electrodes as it is possible to functionalise the CNTs for direct bonding to targeted molecules, thereby preventing non-specific binding or the need of additional bonding layers. Recent advances in chemical functionalisation of CNTs in sensing systems have been reported elsewhere [34-39]. Furthermore, the surface area of a thin film of interconnecting CNTs is significantly greater than the surface area of a metal thin film electrode; hence the binding area is increased without physically increasing the size of the devices. Consequently, for the same concentration of biological samples, a larger number of targeted molecules can be bound onto the resonator's surface compared to devices with metal electrodes, and hence devices with higher sensitivity can be realised.

In the work reported here, AlN-based SMR devices with a fundamental thickness longitudinal mode (TLM) at frequencies around $1.75 \mathrm{GHz}$ were fabricated. A thin film of multi-wall carbon nanotubes (MWCNTs) was directly grown on the AlN film to form the top electrode of the SMR. The frequency response of the devices was measured and the effect of the CNTs electrode was studied by direct comparison of two identical sets of devices, one set having devices with metal electrodes and the second set having devices with top CNTs electrodes. In order to test their suitability as biosensors, protein solutions with different concentrations were loaded on the top of the resonators and their responses to mass-loading from physically adsorbed coatings were investigated.

\section{Experimental}

Two sets of identical SMR devices were fabricated, one set (Set 1) with metal electrodes and another set (Set 2) with an identical metal bottom electrode and a top CNTs electrode. The main steps of the fabrication process are shown in Fig. 2.

The fabrication process commenced with growing five alternating low and high acoustic impedance layers of porous $\mathrm{SiO}_{2}$ and Ir, to form an acoustic reflector centred at $\sim 2 \mathrm{GHz}$ and bandwidth $\sim 1 \mathrm{GHz}$ as shown in Fig. 3, where the one dimension simulation 


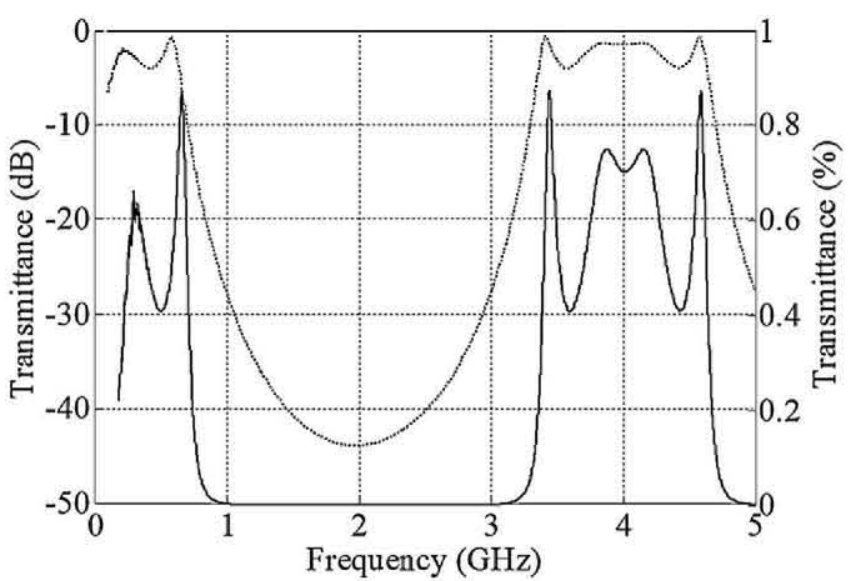

Fig. 3. Simulated transmittance of longitudinal modes on the reflectors fabricated in $\mathrm{AB}$ (dotted line) and \% (solid line).

of the response of the mirror is presented. After polishing the last $\mathrm{SiO}_{2}$ layer of the acoustic reflector to a roughness below $2 \mathrm{~nm}$, a seed layer of Ti/Mo (15-20 nm thick) was used to improve the crystal quality of the Ir bottom electrode and its adhesion to the acoustic reflector. Ir slugs (99.98\% pure) were electron-beam evaporated at a base pressure of less than $1 \times 10^{-6}$ Torr to form bottom electrodes $120 \mathrm{~nm}$ thick. The AlN piezoelectric films were then reactively sputtered from a $150 \mathrm{~mm}$ diameter $\mathrm{Al}$ target in a Leybold Z-550 sputtering system. The AIN films were sputtered with a 3:7 $\mathrm{Ar}: \mathrm{N}_{2}$ admixture at a total pressure of $1.2 \mathrm{mTorr}$, with a pulsed DC power level of $1.2 \mathrm{~kW}$, and a platen temperature of $400^{\circ} \mathrm{C}$. An RF bias was applied to the substrates to tune the stress in the AIN films to $0 \pm 50 \mathrm{MPa}$. These conditions provided deposition rates of $40 \mathrm{~nm} \mathrm{~min}^{-1}$. Before deposition, substrates were degassed close to the AlN deposition temperature. A soft etch process, consisting of a short ( $60 \mathrm{~s}$ ) bombardment with $\mathrm{Ar}^{+}$ions from an $\mathrm{RF}$ glow discharge generated near the substrate, was applied before AlN deposition to enhance $c$-axis orientation and to avoid the growth of tilted microcrystals, related to the degradation of the piezoelectric response [40]. The thickness of the AlN layer was set to $1550 \mathrm{~nm}$.

The crystal quality of the sputtered AIN films was assessed by Xray diffractometry (XRD). The $\theta-2 \theta$ scan of the samples over a broad angle range confirm the high degree of orientation of the films along the $(0002)$ or $c$-axis. This is shown in Fig. 4 where only the peaks corresponding to the $(0002)$ AlN planes, together with those of the (111) Ir planes are observed. The identification of the peaks is based on their tabulated values [41]. The inset of Fig. 4 presents

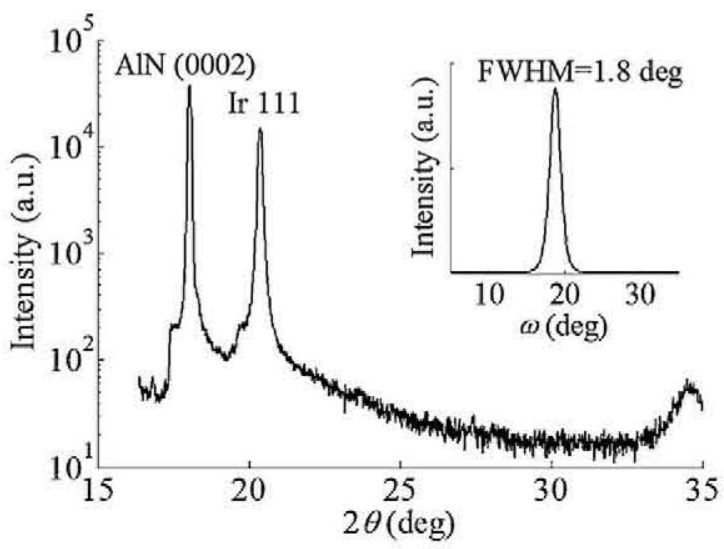

Fig. 4. X-ray diffractogram of a $1.55 \mu \mathrm{m}$-thick $\mathrm{AIN}$ film on a $\mathrm{SiO}_{2} / \mathrm{Ir}$ reflector in a $\theta-2 \theta$ configuration. The inset shows the rocking curve of the AIN $(002)$ peak with a FWHM of $1.8^{\circ}$.

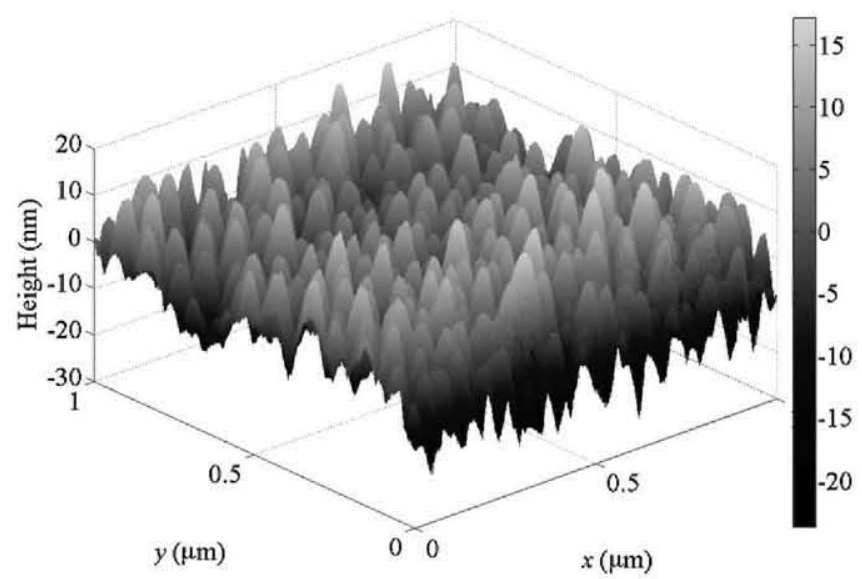

Fig. 5. AFM image of an AIN film AIN film deposited on $\mathrm{SiO}_{2} /$ Ir reflector.

the rocking curve ( $R C$ ) of the most intense reflection from AlN, i.e., the $(0002)$ peak at $\theta=18.02^{\circ}$. The peak has a full width at half maximum (FWHM) of $1.8^{\circ}$, indicating the small angular dispersion of the crystallites around the c-axis.

The surface characteristics of the different layers were inspected by atomic force microscopy (AFM). The roughness of the Ir bottom electrode is around $4 \mathrm{~nm}$ and that of AlN films, shown in Fig. 5, is around $7 \mathrm{~nm}$. Additional information on the polar orientation was obtained by wet chemical etching of the AIN layers. The structure of the AIN surface indicates a $c$-axis orientation of the micro-crystals, as expected from XRD measurements, with a basal plane diameter of around $50 \mathrm{~nm}$.

After AlN deposition, via etch holes were formed through the AlN for electrical connection to the Ir bottom electrode by wet etching the AIN in a warm $\left(\sim 40^{\circ} \mathrm{C}\right) \mathrm{KOH}$ solution through a hard Mo mask, which was subsequently removed at room temperature with a wet etch solution containing phosphoric acid (70\%), nitric acid (10\%), acetic acid blend (10\%) and de-ionised water (10\%). The top electrode was then patterned directly on the AIN film with a lift-off photolithography process. A $10 \mathrm{~nm}$ of $\mathrm{Cr}$ layer followed by a $90 \mathrm{~nm}$ of Au layer was then evaporated on one set of devices (Set 1) to form the top electrode. For the other set of devices (Set 2), a $7 \mathrm{~nm}$ Fe layer, which acts as a catalyst layer for the growth of CNTs, was evaporated with no $\mathrm{Cr} / \mathrm{Au}$ electrode. $\mathrm{CNT}$ growth occurs as a result of the exposure of catalyst nano-particles to a gaseous carbon feedstock at elevated temperatures [30]. The CNTs were directly grown on the second set of the devices by thermal CVD. The substrate containing the patterned Fe catalyst was loaded into the chamber and heated up to $650^{\circ} \mathrm{C}$ at a rate of $4{ }^{\circ} \mathrm{C} \mathrm{min}^{-1}$. Ammonia $\left(\mathrm{NH}_{3}\right)$ was introduced into the chamber to raise the pressure up to $5 \mathrm{mbar}$ and to help maintain the catalyst metal surface activity by reacting with the amorphous carbon. The system was dwelled for several minutes so as to break the catalyst layer into nano-islands, after which the carbon feedstock for CNT growth, acetylene $\left(\mathrm{C}_{2} \mathrm{H}_{2}\right)$, was introduced into the chamber to commence the growth. $\mathrm{C}_{2} \mathrm{H}_{2}$ was introduced into the chamber through a large, flat, stainless steel plate 'showerhead' configuration, which ensures a uniform flow of gas over the whole area of growth. Once the growth is finished, the gases were evacuated and the system was left to cool down. This type of growth produces a forest of interconnecting nanotubes with optimal electrical and mechanical properties [30]. CNTs layers with a thickness of $\sim 100 \mathrm{~nm}$ were grown. The electrical conductivity of these layers was calculated using a four-probe measurement technique and was found to be in the order of $\sim 3.5 \times 10^{5} \mathrm{~S} \mathrm{~m}^{-1}$. This is over an order of magnitude lower than the conductivity of Au, however it is high enough to conduct the low current electrical signal necessary to excite the resonators. 


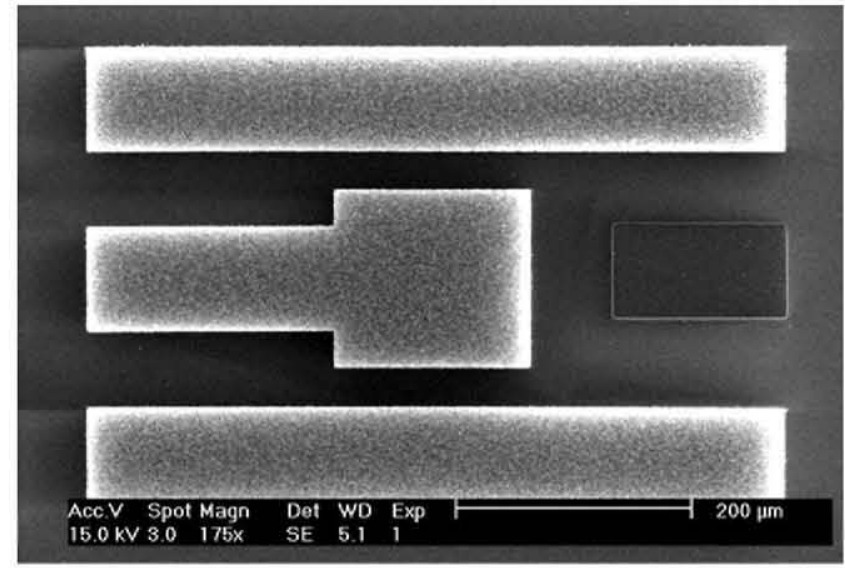

(a)

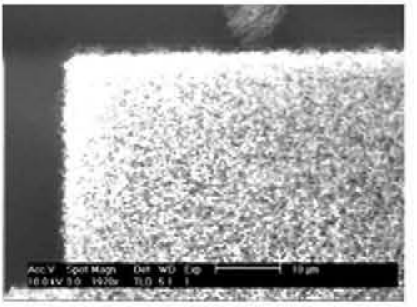

(b)

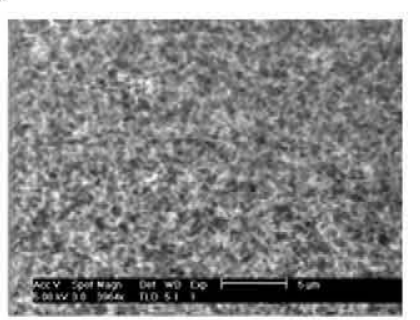

(c)
Fig. 6. (a) SEM image of a fabricated SMR with CNT top electrode, (b) detail of an electrode edge showing excellent definition and (c) top view of the CNTs layer.

Fig. 6 shows an SEM image of a fabricated SMR with CNTs top electrode with detail of the electrode edges showing excellent definition and a top view of the CNTs layer.

The yield of the devices was low $(\sim 20 \%)$ mostly due to the peeling off of the Ir reflector layers during the CNT growth at the high temperatures needed. Yield can be improved by re-optimising the CNTs growth at lower temperatures (although this will lead to an increase in the number of defects on the CNTs) or by the utilisation of a reflector that does not contain metallic layers. Alternatively, CNTs can be grown on an independent substrate and transferred into a liquid solution via an ultrasonic spinner. The solution containing debundled CNTs can then be deposited over the SMR devices with a pipette [29], eliminating the need for high temperature processing.

\section{Results and discussion}

\subsection{SMRs electrical characterisation}

Electrical characterisation of the SMR devices was carried out with a coplanar probe station connected to an Agilent E5062A network analyser. An automated measurement routine was written with LabVIEW ${ }^{\circledR}$ to continuously monitor and record the resonance spectrum of the SMRs. Devices resonating at around $1.75 \mathrm{GHz}$, with resonance spectra that were free from significant spurious modes, were achieved and a typical response of the devices is shown in Fig. 7. It can be seen that the decreased mass associated with the use of the CNT electrodes increased the base resonant frequency of these devices by approximately $80 \mathrm{MHz}$ compared to devices with metal electrodes. It is also observed that the return loss, $R L$, is significantly improved at resonance for the devices with top CNTs electrode. The improvement in the $R L$ magnitude is mostly attributed to the low mass and high acoustic impedance of the CNT electrode, and to the large acoustic losses in Au.

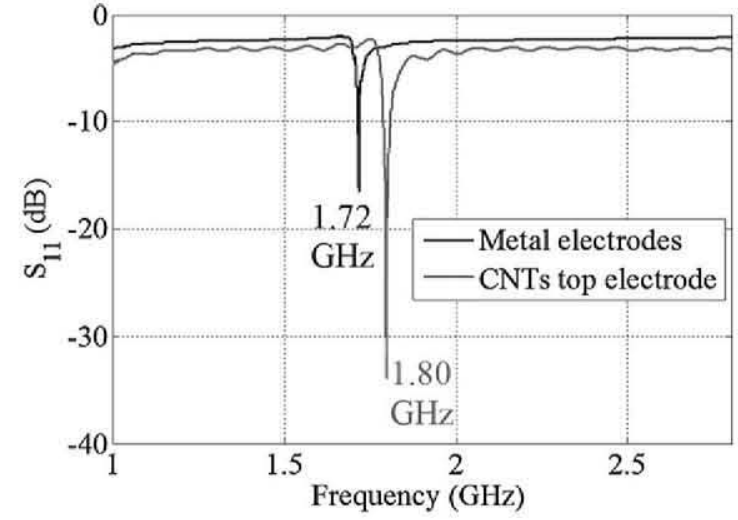

Fig. 7. Typical frequency response of fabricated devices, showing the main resonance in the $1.7-1.8 \mathrm{GHz}$ range.

The quality factor of the antiresonant peak was calculated using the 3-dB method [42] from the equation:

$Q_{3 \mathrm{~dB}}=\frac{f_{\text {ar }}}{\left|f_{2}-f_{1}\right|}$

where $f_{a r}$ is the antiresonant frequency and $f_{2}-f_{1} \mid$ is the bandwidth at $3 \mathrm{~dB}$, both extracted from the S-parameter spectrum.

$Q$ was found to be higher than 2000 for the devices with a CNT electrode and around 1000 for the devices with metal electrodes. Au is malleable and ductile so larger acoustic losses and lower $Q$ are expected in devices with Au electrodes, however it has many benefits for the immobilisation of different biosystems and has previously been successfully used to immobilise antigen/antibodies, proteins and biomarkers [43-46]. Generally devices with higher $Q$ exhibit sharper resonant peaks that allow tracking of a smaller frequency shift. Therefore BAW resonators with a CNTs top electrode have the potential to measure smaller frequency shifts, increasing the sensitivity compared to top metal electrode devices.

Fig. 8(a) shows the excitation bandwidth constrained over $140 \mathrm{MHz}$ near the frequency of resonance of both types of devices. The biggest difference between the resonators with top metal electrodes and those with top CNTs electrode lies on the ripples observed on the resonant peaks. These ripples can be better seen in the Smith chart presented in Fig. 8(b). A similar effect was previously observed on ZnO-based FBARs resonators with CNT electrodes [33]. The ripples are believed to be generated by travelling waves at the surface of the resonators and produce energy losses, and therefore a decrease on $R_{L}$ and the $Q$ factor. The travelling waves are greatly attenuated, although not completely eliminated, by the CNTs electrodes due to the high elastic modulus and discontinuous nature of these compared to the metal electrodes. It is worthwhile noting that these ripples are a well known problem and certain electrode shapes can also help to minimise them $[47,48]$.

\subsection{Comparison of biological sensitivity of devices with metal and CNTs electrodes}

In order to investigate the sensitivity of the devices with top metal and CNTs electrodes, protein solutions with different concentrations were loaded on the top of the resonators and their responses to mass-load from physically adsorbed coatings were calculated following the steps summarised in Fig. 9.

Firstly the resonant frequency of unloaded resonators was tracked over a period of $\sim 30 \mathrm{~min}$ and was checked to be stable with changes of less than $2 \mathrm{kHz}$. Bovine Serum Albumin (BSA) solutions in phosphate buffer (ionic strength $=20 \mathrm{mM}$ and $\mathrm{pH}=7$ ) with different concentrations were then placed on the top of different sets of identical SMRs both with top metal and CNTs 

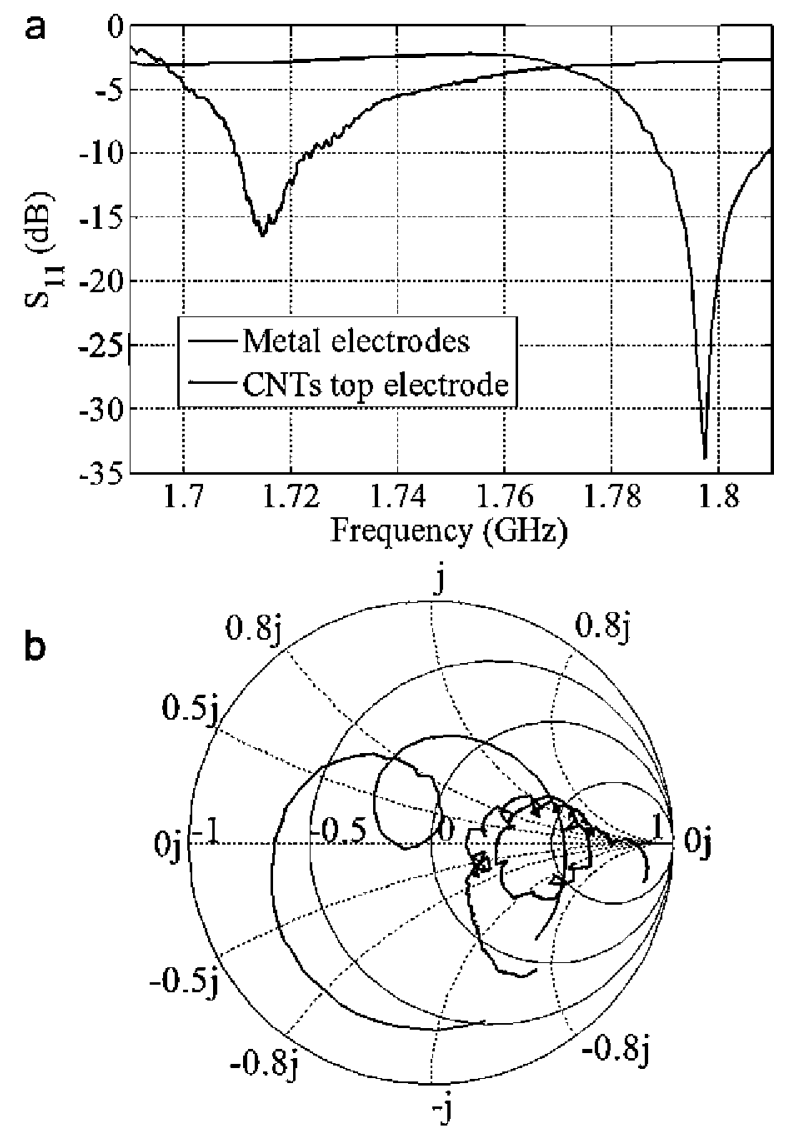

Fig. 8. (a) Excitation bandwidth constrained over $140 \mathrm{MHz}$ showing the main resonance peak of fabricated devices and (b) Smith chart of the return loss.

electrodes, and allowed them to physically adsorb onto the SMRs' surface for $15 \mathrm{~min}$, after which they were rinsed with a buffer and dried with $\mathrm{N}_{2}$. Measurements were subsequently carried out on loaded devices when they were completely dried. Their resonant frequency was again tracked over a period of $\sim 30 \mathrm{~min}$ and was checked to be stable. A comparison of $f_{r}$ of loaded and unloaded resonators yields the frequency shift $\Delta f_{r}$, and the results are shown in Fig. 10 as a function of BSA concentration.

The frequency shift was of the order of hundreds of $\mathrm{kHz}$ in both cases but the absolute frequency changes observed on the SMRs with CNTs electrodes are significantly greater than those with metal electrodes. The sensitivity ( $\triangle f_{r} /$ BSA concentration) of devices with CNTs top electrode is $\sim 3.4 \mathrm{kHz} \mathrm{mg}^{-1} \mathrm{l}^{-1}$ whilst the sensitivity of devices with metal electrodes is $\sim 1.6 \mathrm{kHz} \mathrm{mg}^{-1} \mathrm{l}^{-1}$.

Numerical simulations of the sensitivities of the fabricated devices to BSA were carried out by using the one dimension

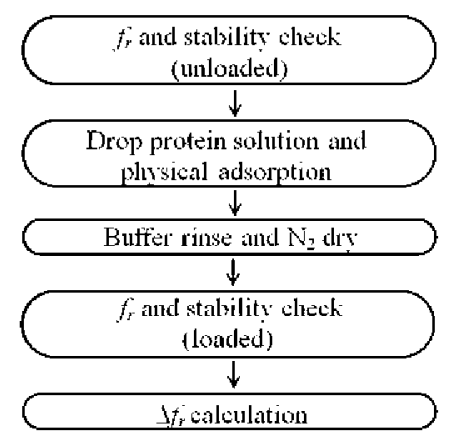

Fig. 9. Mass load responses measurement: main steps.

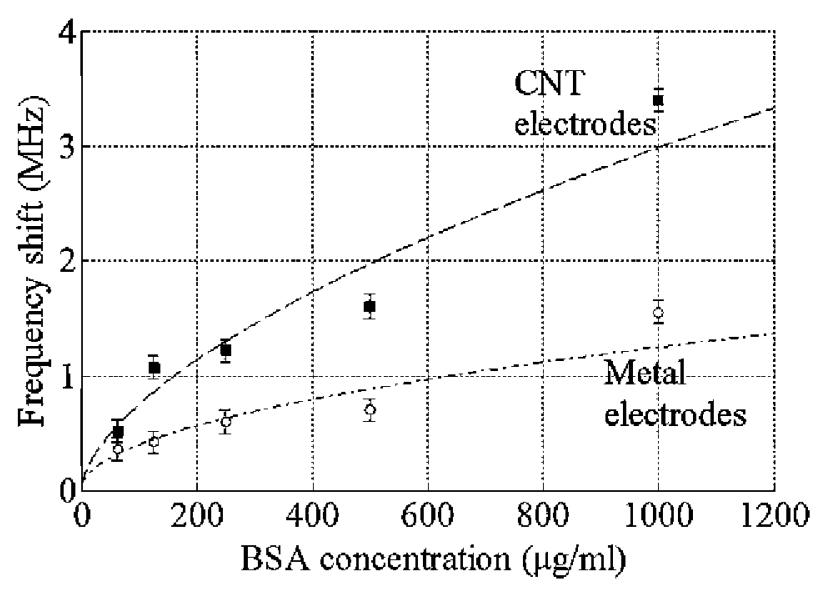

Fig. 10. Frequency shift observed as a function of BSA concentration. The dashed lines are a guide for the eye.

Mason's model [49]. Thin layers of BSA proteins (density $\sim 1 \mathrm{~g} \mathrm{~cm}^{-3}$ [50]) were numerically added on top of both resonators and their responses to the additional mass loading were calculated as shown in Fig. 11. A linear relationship is obtained in both types of resonators for the small additional mass loading range studied. Devices with CNTs top electrodes are significantly more sensitive $\left(0.25 \mathrm{MHz} \mathrm{cm}^{2} \mathrm{ng}^{-1}\right)$ than devices with metallic electrodes $\left(0.14 \mathrm{MHz} \mathrm{cm}^{2} \mathrm{ng}^{-1}\right)$ due to the lower pre-mass loading effect introduced by the electrodes (a CNTs electrode is nearly 20 times lighter than a $\mathrm{Cr} / \mathrm{Au}$ electrode). Both these sensitivities to BSA are over three orders of magnitude larger than that previously reported of QCMs [51] and considerably greater than other types of sensors [52,53].

From the sensitivity values calculated theoretically, the effective protein mass loading that occurs from physically adsorbing BSA on Au and CNTs can be calculated, and it is shown in Fig. 12. The effective BSA mass binding onto the resonators' surface does not follow a linear dependence with the BSA concentration in the solution. This is a well known saturation effect (see for example [54]) where excess of protein restricts access to the binding surface. As seen in Fig. 12, the aforementioned saturation effect occurs at lower protein concentrations for devices with metallic electrodes compared to devices with CNT electrodes due to the smaller binding area of a flat electrode compared to the CNTs electrode.

It must also be noticed that although a higher effective mass loading is occurring on the CNTs electrodes, this is not as high as

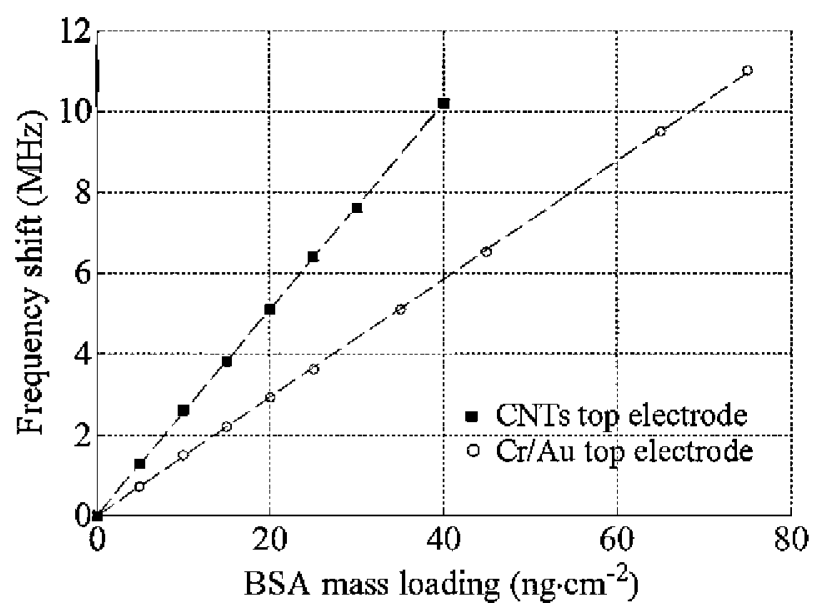

Fig. 11. Numerical simulations of the sensitivities to BSA of the fabricated SMRs with $\mathrm{Cr} / \mathrm{Au}$ and $\mathrm{CNTs}$ top electrodes. 


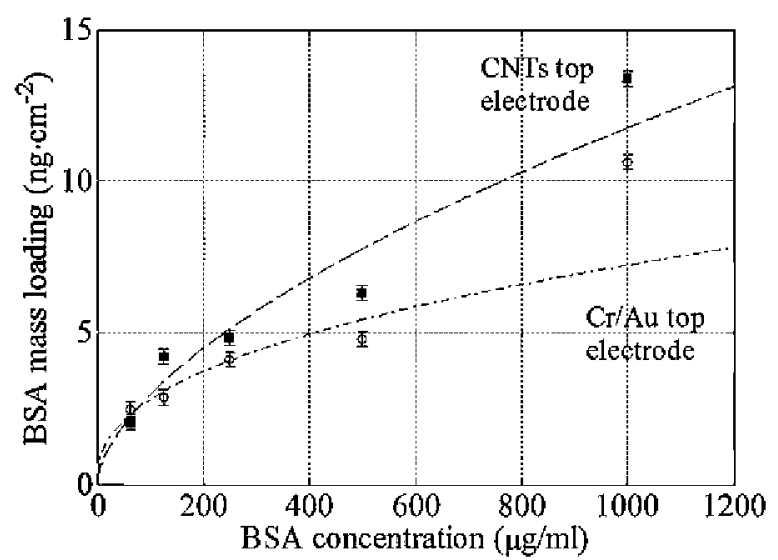

Fig. 12. Effective BSA mass loading on the resonators' surface for different BSA concentrations. The dashed lines are a guide for the eye.

one would expect from a binding area which is around one order of magnitude greater than that of a flat metal. This confirms the lower binding rate of proteins to CNTs compared than to Au, and highlights the need of functionalising CNTs through chemical treatment before protein adsorption.

\section{Conclusions}

AlN-based SMR with a CNTs top electrode have been shown to have many advantages for the development of acoustic wave based biosensors. The CNTs were directly grown on the AlN surface by thermal CVD, and their influence on the electro-acoustic response of the resonators was assessed by direct comparison of two identical sets of resonators; one set comprised of devices with metal electrodes and the second set comprised with resonators with a top CNTs electrode. Devices resonating at around $1.75 \mathrm{GHz}$ were achieved and exhibited a significantly large unloaded quality factor $Q>2000$, which is much greater than the identical devices fabricated with metal electrodes. This is attributed to a combination of lower acoustic losses on CNTs electrodes compared to gold electrodes, lower density of the CNTs electrodes (hence lower mass) and higher acoustic impedance of the CNTs electrodes which contributes to a higher energy trapping within the piezoelectric. Devices with higher $Q$ factor possess higher sensitivity due to sharper resonance peaks which facilitates tracking smaller frequency shifts. Bovine serum albumin protein solutions with different concentrations were loaded on the top of the resonators and their responses to mass-load from physically adsorbed coatings were investigated. It was found that resonators using CNTs as the top electrode material exhibited higher frequency change for a given load due to the higher sensitivity $\left(0.25 \mathrm{MHz} \mathrm{cm}^{2} \mathrm{ng}^{-1}\right)$ of these devices compared to that of devices with metallic electrodes $\left(0.14 \mathrm{MHz} \mathrm{cm}^{2} \mathrm{ng}^{-1}\right)$. However the sensitivity of devices with a top CNTs electrode is not as high as expected from devices possessing an effective binding area around 1 order of magnitude greater, indicating that the protein binding to carbon is not well optimised as for gold. Thus future work should concentrate on the chemical functionalisation of CNTs for improving the direct bonding of targeted molecules, which will in turn further increase the sensitivity of these biosensors.

\section{Acknowledgements}

The work reported here was supported by the EPSRC, grant numbers EP/F063865/1, EP/F06294X/1, EP/F062966/1 and EP/E023614/1. Iborra, Clemet and Olivares acknowledge financial support from the Ministerio de Ciencia e Innovación of Spain through project MAT2010-18933, and from the European Union through the European Regional Development Fund (FEDER).

\section{References}

[1] B.P. Nelson, T.E. Grimsrud, M.R. Liles, R.M. Goodman, R.M. Corn, Surface plasmon resonance imaging measurements of DNA and RNA hybridization adsorption onto DNA microarrays, Anal. Chem. 73 (1999) 1-7.

[2] E.P. Furlani, R. Biswas, A.N. Cartwright, N.M. Litchinitser, Antiresonant guiding optofluidic biosensor, Opt. Commun. 284 (2011) 4094-4098.

[3] Z. Zhu, W. Song, K. Burugapalli, F. Moussy, Y. Li, X. Zhong, Nano-yarn carbon nanotube fiber based enzymatic glucose biosensor, Nanotechnology 21 (2010) 165501.

[4] J.A. Lee, S. Hwang, J. Kwak, S. Park, S.S. Lee, K.-C. Lee, An electrochemical impedance biosensor with apatamer modified pyrolyzed carbon electrode for label-free protein detection, Sens. Actuators B: Chem. 129 (2008) 372-379.

[5] M.J. Oliver, J. Hernando-Garcia, A. Ababneh, H. Seidel, U. Schmid, J. Olivares, E. Iborra, P. Pobedinskas, K. Haenen, J.L. Sanchez-Rojas, Resonant piezoelectric AlN-actuated microcantilevers for detection of antigen/antibody interactions, Proc. SPIE (2011) 80661A.

[6] R. Raiteri, M.Grattarola, H.-J. Butt, P. Skládal, Micromechanical cantilever-based biosensors, Sens. Actuators B: Chem. 79 (2001) 115-126.

[7] C. Zerrouki, N. Fourati, M. Lazerges, J.M. Fougnion, C. Vedrine, C. Pernelle, Shear horizontal surface acoustic waves sensor for label-free DNA detection, Sens. Lett. (2010) 813-817.

[8] H. Zhang, W. Pang, M.S. Marma, C.-Y. Lee, S.K. Bahl, E.S. Kim, C.E. McKenna, Label-free detection of protein-ligand interactions in real time using micromachined bulk acoustic resonators, Appl. Phys. Lett. 96 (2010) 123702.

[9] R. Ruby, Review and comparison of bulk acoust ic wave FBAR, SMR technology, IEEE Ultrason. Symp. 102 (2007) 9-1040.

[10] G. Sauerbrey, Erwendung von schwingqarzen zur wagung dunner schichten un zur mikrowagung, Z. Physik 20 (1959) 6-222.

[11] A. Shons, J. Najarian, F. Dorman, Immunospecific microbalance, J. Biomed. Matter. Res. 6(1972) 565-570.

[12] K.M. Lakin, S.J. Wang, Acoustic bulk wave composite resonators, Appl. Phys. Lett. 38 (1981) 125-127.

[13] T. Nishihara, T. Yokoyama, T. Miyashita, Y. Satoh, High performance and miniature thin film bulk acoustic wave filters for $5 \mathrm{GHz}$, IEEE Ultrason. Symp. 96 (2002) 9-972.

[14] R.C. Ruby, P. Bradley, Y. Oshmyansky, A. Chien, J.D. Larson III, Thin film bulk wave acoustic resonators (FBAR) for wireless applications, IEEE Ultrason. Symp. 81 (2001) 3-821.

[15] M.A. Dubois, J.F. Carpentier, P. Vincent, C. Billard, G. Parat, C. Muller, P. Ancey, P. Conti, Monolithic above-IC resonator technology for integrated architectures in mobile and wireless communication, IEEE J. Solid State Circ. 41 (2006) 7-16.

[16] P-H. Sung, C-M. Fang, P.-Z. Chang, Y.-C. Chin, P.-Y. Chen, The method for integrating FBAR with circuitry on CMOS chip, IEEE Ultrason. Symp. 56 (2004) 2-565.

[17] M.L. Johnston, I. Kymissis, K.L. Shepard, FBAR-CMOS oscillator array for masssensing applications, IEEE Sens. J. 10 (2010) 1042-1047.

[18] J.Weber, W.M. Albers, ]. Tuppurainen, M. Linka, R. Gabla, W. Wersinga, M. Schre itera, Shear mode FBARs as highly sensitive liquid biosensors, Sens. Actuators A: Phys. 128 (2006) 84-88.

[19] G. Wingqvist, J. Bjurston, L. Liljeholm, V. Yantchev, I. Katardjiev, Shear mode AlN thin film electro-acoustic resonant sensor operation in viscous media, Sens. Actuators B: Chem. 123 (2007) 466-473.

[20] M. Nirsch, M. Schreiter, J. Vörös, Comparison of FBAR and QCM-D sensitivity dependence on adlayer thickness and viscosity, Sens. Actuators A: Phys. 165 (2011) 415-421.

[21] P.B. Kirby, M.D.G. Potter, C.P. Williams, M.Y. Lim, Thin film piezoelectric property considerations for surface acoustic wave and thin film bulk acoustic resonators, J. Eur. Ceram. Soc. 23 (2003) 2689-2692.

[22] E. Iborra, M. Clement, J. Capilla, J. Olivares, V. Felmetsger, Optimization of thin AIN sputtered films for $\mathrm{X}$-band BAW resonators, IEEE Ultrason. Symp. 168 (2010) 8-1691.

[23] G. Ferblantier, F. Mailly, R. Asmara, A. Foucarana, F. Pascal-Delannoya, Deposition of zinc oxide thin films for application in bulk acoustic wave resonator, Sens. Actuators A: Phys, 122 (2005) 184-188.

[24] S-H. Choi, J-S. Kim, Study on the c-axis preferred orientation of ZnO film on various metal electrodes, Ultramicroscopy 108 (2008) 1288-1291.

[25] R. Harta, E. Ergezena, R. Leca, H. Noh, Improved protein detection on an AC electrokinetic quartz crystal microbalance (EKQCM), Biosens. Bioelectron 26 (2011) 3391-3397.

[26] L. García-Gancedo, J. Pedrós, A.J. Flewitt, W.I. Milne, G.M. Ashley, J.K. Luo, C.J.B. Ford, Ultrafast sputtered ZnO thin films with high $k_{T}$ for acoustic wave device applications, IEEE Ultrason. Symp. 106 (2010) 4-1067.

[27] T. Yokoyama, T. Nishihara, S. Taniguchi, M. Iwaki, Y.Satoh, M. Ueda, T. Miyashita, New electrode material for low-loss and high Q FBAR filters, IEEE Ultrason. Symp. 42 (2004) 9-432.

[28] S. Tanifuji, Y. Aota, H. Oguma, S. Kameda, T. Takagi, K. Tsubouchi, Spurious vibration suppression by film thickness control FBAR, IEEE Ultrason. Symp. 219 (2008) 3-2196.

[29] M. Dragoman, A. Muller, D. Neculoiu, G. Vasilache, K. Konstantinidis, D. Grenier, L. Dubuc, R. Bary, R. Plana, E. Flahaut, High performance thin film bulk acoustic resonator covered with carbon nanotubes, Appl. Phys. Lett. 89 (2006) 143122. 
[30] M. Mann, Y. Zhang, K.B.K. Teo, T. Wells, M.M. El-Gomati, W.I. Milne, Controlling the growth of carbon nanotubes for electronic devices, Microelectron. Eng. 87 (2010) 1491-1493.

[31] L. Garcia-Gancedo, F. Al-Naimi, A.J. Flewitt, W.I. Milne, G.M. Ashley, J.K. Luo, X.B. Zhao, J.R. Lu, Fabrication of high-Q film bulk acoustic resonator (FBAR) filters with carbon nanotube (CNT) electrodes, IEEE Ultrason. Symp. 30(2010) 1-304

[32] T. Xu, Z. Wang, J. Miao, Fabrication of carbon-nanotube enhanced piezoelectric membrane-based biosensor, IEEE Int. Nano. Conf. Proc. 55 (2008) 5-558.

[33] L. García-Gancedo, F. Al-Naimi, A.J. Flewitt, W.I. Milne, G.M. Ashley, J.K. Luo, X.B. Zhao, J.R. Lu, ZnO-based FBAR resonators with Carbon Nanotube (CNT) electrodes, IEEE Trans. Ultr. Fer. Freq. Cont. 58 (2011).

[34] S. Liu, Q. Shen, Y. Cao, L. Gan, Z. Wang, L. Steigerwald, X. Guo, Chemical functionalization of single-walled carbon nanotube field-effect transistors as switches and sensors, Coord. Chem. Rev. 254 (2010) 1101-1116.

[35] A.Star, J.P. Gabriel, K. Bradley, G. Grüner, Electronic detection of specific protein binding using nanotube FET devices, Nanoletters 3 (2003) 459-463.

[36] P. Qi, O. Vermesh, M. Grecu, A. Javey, Q, Wang, H. Dai, Toward large arrays of multiplex functionalized carbon nanotube sensors for highly sensitive and selective molecular detection, Nanoletters 3 (2003) 347-351.

[37] J.F. Lu, L. Gu, M.J. Meziani, X. Wang, P.G. Luo, L.M. Veca, L. Cao, Y.-P. Sun, Advances in bioapplications of carbon nanotubes, Adv. Mater. 21 (2009) $139-151$.

[38] A. Modi, N. Koratkar, E. Lass, B. Wei, P.M. Ajayan, Miniaturized gas ionization sensors using carbon nanotubes, Nature 424 (2003) 171-174

[39] J. Li, A.M. Cassell, H. Dai, Carbon nanotubes as AFM tips: Measuring DNA molecules at the liquid/solid interface, Surf. Interface Anal. 28 (1999) 8-11.

[40] M. Clement, J. Olivares, E. Iborra, S. González-Castilla, N. Rimmer, A. Rastogi, AlN films sputtered on iridium electrodes for bulk acoustic wave resonators, Thin Solid Films 517 (2009) 4673-4678.

[41] ICDD Powder-Diffraction-File (PDF) 25-1133.

[42] K.J. Coakley, J.D. Splett, M.D. Janezic, R.F. Kaiser, Estimation of Q factors and resonant frequencies, IEEE Trans. Micro. Theo. Tech. 51 (2003) 862868.

[43] T. Ibii, M. Kaieda, S. Hatakeyama, H. Shiotsuka, H. Watanabe, M. Umetsu, I. Kumagai, T. Imamura, Direct immobilization of gold-binding antibody fragments for immunosensor applications, Anal. Chem. 82 (2010) 42294235 .

[44] A.A. Karyakin, G.V. Presnova, M.Y. Rubtsova, A.M. Egorov, Oriented immobilization of antibodies onto the gold surfaces via their native thiol groups. Anal Chem. 72 (2000) 3805-3811

[45] K. Won, M.-J. Park, H. Yoon, J.H. Kim, Immobilization of iron storage protein on a gold electrode based on self-assembled monolayers, Ultramicroscopy 108 (2008) 1342-1347.

[46] E. Casero, L. Vázquez, ]. Martín-Benito, M.A. Morcillo, E. Lorenzo, F. Pariente, Immobilization of Metallothionein on gold/mica surfaces: relationship between surface morphology and protein-substrate interaction, Langmuir 18 (2002) 5909-5920.

[47] D. Rosen, J. Bjurstom, I. Katardjiev, Suppression of spurious lateral modes in thickness-excited FBAR resonators, IEEE Trans. Ultr. Fer. Freq. Cont. 52 (2005) $1189-1192$.

[48] S. Tanifuji, Y. Aota, H. Oguma, S. Kameda, T. Takagi, K. Tsubouchi, Spurious vibration suppression by film thickness control for FBAR, IEEE Ult rason. Symp. $219(2008) 3-2196$

[49] J. Rosenbaum, Bulk Acoustic Waves Theory and Devices, Dec. 1988, Artech House, Boston, 1988 (reprint)

[50] A. Jonas, Physicochemical properties of bovine serum high density lipoprotein, J. Biol. Chem. 247 (1972) 7767-7772.

[51] Q. Xie, Y.Zhang, M. Xu, Z. Li, Y. Yuan, S. Yao, Combined quartz crystal impedance and electrochemical impedance measurements during adsorption of bovine serum albumin onto bare and cysteine- or thiophenol-modified gold electrodes, J. Electroanal. Chem. 478 (1999) 1-8.

[52] X. Sun, B. Liu, F. He, A novel biosensor for bovine serum albumin based on fluorescent self-assembled sandwich bilayer, Luminescence 24 (2009) 62-66.

[53] M.I. Zibaii, H. Lafiti, M. Arabsorkhi, A. Kazemi, M. Gholami, M.K. Azar S.M. Hosseini, Biconical tapered optical fiber biosensor for real-time monitoring of bovine serum albumin at femtogram $/ \mathrm{mL}$ levels on antibodyimmobilized tapered fibers, Proc. SPIE: Int. Soc. Opt. Eng. 7653 (2010) 765322.

[54] X. Zhao, F. Pan, B. Cowsill, J.R. Lu, L. Garcia-Gancedo, A.J. Flewitt, G.M. Ashley, J.K. Luo, Interfacial immobilization of monoclonal antibody and detection of human prostate-specific antigen, Langmuir 27 (2011) 7654-7662.

\section{Biographies}

Luis García-Gancedo received a B.Sc. in physics from the University of Oviedo, Spain in 2003. He then joined the University of Brighton (UK) where he received a Ph.D. for his work on magnetostrictive ultrasonic transducers for SONAR applications. After completing his Ph.D., Luis worked as a Research Fellow at the University of Birmingham (UK), in a multidisciplinary project fabricating ultrasonic transducers and arrays for ultrahigh resolution real time biomedical imaging. He joined Cambridge University (UK) in January 2009, and he is at present a Research Associate at the Electrical Engineering Division, working on the development of thin film bulk acoustic resonators (FBARs) based ultrahigh sensitive biosensor arrays for diagnostic applications. Since October 2010 Luis is also a Lecturer in Engineering at Newnham College, University of Cambridge.

Zhigang Zhu received a Ph.D. degree in Shanghai Institute of Ceramics, Chinese Academy of Sciences (2005), where he worked on functional materials and devices. After that, he was a Royal Society International Research Fellow in the Micro-Engineering and Nanotechnology Research Centre at the University of Birmingham (2005-2006), working on micro-scale ceramic components by soft lithography. He then moved to London and spent two and half years in Brune University as a Research Fellow (2007-2009), focused on the implantable glucose biosensors. At the moment, he is a Research Associate at the University of Cambridge working in MEMS (Micro-Electro-Mechanical Systems) design and fabrication for biosensors. He has published over 20 peer-reviewed journal papers and overall citations are nearly 100 times. His main research interests includes MEMS, implantable biosensors, CNT based biosensors, functional materials and devices.

Enrique Iborra received the M.Sc. degree in physics from the Universidad Complutense de Madrid, Madrid, Spain, in 1982. He received the Ph.D. degree (with distinction) in physics from the same university in 1986. In 1987, he joined the Department of Electronic Technology at the Universidad Politecnica de Madrid, Madrid, Spain, as Associate Professor. Now, he is Full Professor in the same depart ment. Since 1995 he has been the head of the Group of Mic rosystems and Electronic Materials (GMME) in the Department of Electronic Technology at the Polytechnic University of Madrid (UPM). He has been working in thin film electronic materials and sensor devices since 1982. His current research interests are the applications of piezoelectric AIN thin films to SAW, BAW, and RF-MEMS devices.

Marta Clement received the M.Sc. degree in physics from the Universidad Com plutense de Madrid, Madrid, Spain, in 1985. She received the Ph.D. degree in applied physics from the Universidad Autonoma de Madrid, Madrid, Spain, in 1990, for research on metal silicide contacts on gallium arsenide. She spent a year at AT\&T Microelectronics in Madrid working as process engineer in the plasma area. In 1991, she joined the Department of Electronic Technology at the Universidad Politecnica de Madrid, Madrid Spain, as an Associate Professor. From 1991 to 1998 she was involved in the development of electronic materials for infrared sensor applications. Her current interest is the application of polycrystalline AIN films in acoustic resonators (SAW and BAW) and piezoelectric based MEMS.

Jimena Olivares received the B.Sc. degree and the M.Sc. degree in physics from the Universidad Autonoma de Madrid, Madrid, Spain, in 1995 and 1997, respectively. She received the Ph.D. degree in physics from the Universidad Politecnica de Madrid, Madrid Spain, in 2001 for a research work on polycrystalline SiGe thin films. She spent 2 years in a Spanish company, where she worked in the field of technology transfer and in the management of European research projects. In 2003, she joined the Group of Microsystems and Electronic Materials of the Department of Electronic Technology of the Universidad Politecnica de Madrid. Now, she is an Associate Professor in the same department. Her current research interests are surface and bulk micromachining for piezoelectrically based MEMS applications.

Andrew J. Flewitt received the B.Sc. degree in physics from the University of Birmingham, Birmingham, U.K., in 1994 and the Ph.D. degree in scanning tunneling microscopy of amorphous silicon from the University of Cambridge, Cambridge, U.K., in 1998. Following this, he was a Research Associate studying the low-temperature growth of silicon-based materials in the Engineering Department, University of Cambridge. He was appointed to a Lectureship in the same Department in 2002 Since 2009, he has held the position of University Reader in Electronic Engineering His research interests span a broad range of large area electronics and related fields, including thin film transistors and MEMS devices. Dr. Flewitt is a Chartered Physicist and a Member of the Institute of Physics and the Institution of Engineering and Technology.

William I. Milne, FREng FIET, FIMMM, has been Head of the Electrical Enoineering Division at Cambridge University since 1999 and Director of the Centre for Advanced Photonics and Electronics (CAPE) since 2004. He obtained his B.Sc. from St Andrews University in Scotland in 1970 and then went on to read for a Ph.D. in Electronic Materials at Imperial College London. He was awarded his Ph.D. and DIC in 1973 and in 2003, a D.Eng. (Honoris Causa) from University of Waterloo, Canada. He was elected as Fellow of the Royal Academy of Engineering in 2006 and was awarded the II Thomson medal from the IET in 2008. He is a Guest Professor at HuangZhou University in Wuhan, China and a Distinguished Visiting Professor at SEU in Nanjing China and at NUS, Singapore in 2010. He is also a Distinguished Visiting Scholar at KyungHee University, Seoul. He has published/presented $\sim 750$ papers, of which 150 were invited.

Gregory M. Ashley received the B.Sc. degree in microbiology from the University of Liverpool in 1996, then the M.Sc. degree in Biosensors from the University of Manchester in 2000. After this, he went on to obtain a Ph.D. in Biosensors in the University of Cranfield with an emphasis on RF MEMS based Biosensors. He is at present a Postdoctoral Research Fellow at the University of Bolton, working on FBAR based biosensors.

Jack Luo received his Ph.D. from the University of Hokkaido, Japan in 1989. He worked in Cardiff University as a research fellow, in Newport Wafer Fab. Ltd., Philips Semiconductor $\mathrm{Co}$. and Cavendish Kinetics Ltd. as an engineer, senior engineer and manager, and then in Cambridge University as a senior researcher from 2004. From January 2007, he became a Professor in MEMS at the Centre for Material Research and Innovation (CMRI), University of Bolton. His current research interests focus on 
microsystems and sensors for biotechnology and healthcare applications, and third generation thin film solar cells using novel low cost materials.

XiuBo Zhao was born in Jiangsu, China in Feb. 1980. In 2006, he received his Ph.D. degree in biological physics from the University of Manchester. He is currently working at the University of Manchester as an EPSRC funded postdoctora research associate. His current research interests include biomarker immobilisa tion and detection for biosensoring; peptide self-assembly; non-viral gene delivery: interfacial adsorption of biomolecules; biomaterials and surface biocompatibility etc. He is a member of the Institute of Physics, UK. He has authored or co-authored more than 40 peer reviewed papers and 3 book chapters.
Jian R. Lu is a Professor of biological physics, and head of the Biological Physics Group at the University of Manchester. He obtained his Ph.D. degree from Hull University in 1991 in surface chemistry and went on a post-doctoral fellowship in Physical and Theoretical Chemistry Laboratory at Oxford University. From 1995 to 2000 he worked as a Lecturer and Reader at Surrey University before taking the chair at Manchester in 2001 . He has developed an international reputation in the use of neutron reflection and related techniques for studying peptide and protein adsorption. 